Excellence in emergency doctors

\title{
In search of excellence
}

\section{G Hughes}

n Search Of Excellence, by Tom Peters and Robert Waterman, ${ }^{1}$ published in 1982, remains one of the biggest selling and most widely read business books ever. It was then, and still is, touted as a seminal text for those trying to improve systems and deliver quality to the public, mainly in the context of business. I was advised to read it on a senior registrar management course I attended in 1989. It's not a page-turning bodice-ripper. I didn't finish it. I tried hard.

I mention this because I came across it the other day while perusing the shelves of the hospital library. Later that day I discussed the book with a friend who runs a successful business. He agreed that it is still widely referenced in business and training seminars. He then asked me the killer question "what are you guys [emergency doctors] excellent at?" He followed this up by asking "don't you refer everything on to specialists?". I was well behaved and refrained from pouring a jug of water over him.

So, what are we excellent at? By the nature of our work, we are generalists and are pretty good at many things, but what are we excellent at? In an era that revels in having "top-10" lists for virtually everything, here are 10 things we are or should be excellent at. I'm sure there will be disagreement with my selection.

1. Resuscitation: This is the "raison d'etre" for our existence. If we don't do this better than anyone else, then we need to shut the shop and throw away the keys. Knowledge of protocols, ability to think 2-3 steps ahead in decision making and excellent team leadership should be par for the course.

2. Resuscitation procedures: Apart from basics such as the correct way to maintain an airway, cannulate, defibrillate and perform external cardiac massage, we need to be excellent at rapid sequence induction and intubation, recognition and management of the difficult airway, advanced cannulation (be it central or peripheral, arterial or venous) and intercostal drain insertion. We need to perform to the same standard as our anaesthetic and intensivist colleagues.
Knowledge of basic ventilators and ventilation modalities is a prerequisite too.

3. Triage and major incident management: This is core business. As with resuscitation, it is part of our armamentarium. These are not easy skills to acquire. We can be proud of our abilities in this specialty, but we can always do it better.

4. Imaging: From time to time we will always need the skills of a radiologist to help us interpret films, whatever the radiological modality. A skill we can be excellent at is the use of diagnostic ultrasound in the resuscitation room. Focused assessment with sonography for trauma scanning, searching for abdominal aortic aneurysms in the appropriate context and looking for a dilated right ventricle (via an echocardiogram) in massive pulmonary embolism can be core skills. Ultrasound also improves the safety of central cannulation via the internal jugular route. It goes without saying that fracture recognition on plain film is a key skill.

5. Electrocardiograms: After measuring vital signs, the glucose level, and carrying out urine analysis, recording an electrocardiogram is one of our most common investigations. In addition to recognising arrhythmias, all degrees of heart block, and the various patterns seen in (or mimicking) acute coronary syndrome and oddities, such as J waves in hypothermia, we need to be good enough to pick up rare and subtle abnormalities which can have a devastating outcome if missed, such as long QT syndrome and tri-fascicular block.

6. Wounds and soft tissue infections problems: This is core business. In addition to managing everyday problems with skill and precision, we need to promptly recognise occasional conditions such as erythema nodosum, necrotising fasciitis and so forth. Our accuracy for appropriate and timely referral to surgical specialties for both wounds and infections needs to be spot on.
7. Analgesia and conscious sedation: Managing pain is a fundamental part of our work. Although it becomes a heart sink problem when faced with the frequent flyer attendee who has intractable chronic pain that even the pain clinic has given up on, for the rest, speedy and appropriate analgesia is critical. Whether we give it orally, rectally, intranasally, parenterally or via a regional block, we should offer the service with expertise and timeliness. Conscious sedation for minor procedures, with a benzodiazepine and an opiate or a neuroleptic, should have a safety record second to none.

8. Communication: For many reasons, sadly one of them being medicolegal, our record keeping, be it electronic or hard copy, needs to be faultless. We need to set an example for everyone else. Our oral and written abilities to communicate with each other in a department, a hospital, our colleagues in the community and pre-hospital arenas and, last but not least, with our patient and their carers should be exemplary.

9. Acting rationally to the unexpected and unknown, thinking on our feet and keeping calm in a crisis: What more is there to say?

10. Empathy and compassion: Let's show to the patient and their carers that we care. Among the hurly-burly of our work, we must remember that there is more to what we do than intellectual thought, professional achievement and diagnostic precision. In an age driven by targets, instant gratification and materialism, we need to recall why most of us entered this challengingly compelling profession. Most of our clientele come to us at a moment of dire need. For those who die, the way we manage the early grief and bereavement of their relatives and friends is critical. Let's maintain our humanity and compassion and protect their dignity.

Emerg Med J 2006;23:822

doi: $10.1136 /$ emj.2006.041673

Correspondence to: Geoff Hughes, The Emergency Department, Royal Adelaide Hospital, North Terrace, Adelaide 5000, Australia; cchdhb@yahoo.com

Accepted 29 August 2006

Competing interests: None declared.

\section{REFERENCE}

1 Peters T, Waterman R. In search of excellence. 1982. 